\title{
Tau Radiative Decays in the Light Front Quark Model
}

\author{
C. Q. Geng and C. C. Lih \\ Department of Physics, National Tsing Hua University \\ Hsinchu, Taiwan, Republic of China
}

\begin{abstract}
We study the decays of $\tau^{-} \rightarrow \nu_{\tau} P^{-} \gamma\left(P=\pi^{-}, K^{-}\right)$in the light front quark model. We calculate the form factors and use them to evaluate the decay widths. We find that, in the standard model, the decay widths are $1.62 \times 10^{-2}\left(3.86 \times 10^{-3}\right) \Gamma_{\tau^{-} \rightarrow \nu_{\tau} \pi^{-}}$ and $1.91 \times 10^{-3}\left(5.38 \times 10^{-4}\right) \Gamma_{\tau^{-} \rightarrow \nu_{\tau} K^{-}}$with the cuts of $E_{\gamma}=50(400) \mathrm{MeV}$ and $t_{0}=$ 800 (1200) MeV for $\tau^{-} \rightarrow \nu_{\tau} \pi^{-} \gamma$ and $\tau^{-} \rightarrow \nu_{\tau} K^{-} \gamma$, respectively. We also show that with including the radiative decay widths, the experimental rate for $\tau^{-} \rightarrow \nu_{\tau} P^{-}$can be explained.
\end{abstract}




\section{Introduction}

Tau is the only charged lepton which decays into hadrons. Theoretically, the hadronic $\tau$ decays can provide us with valuable information on strong interaction. The simplest rare radiative $\tau$ decays are $\tau^{-} \rightarrow \nu_{\tau} P^{-} \gamma$ with $P$ being the pseudoscalar mesons of $\pi$ and $K$. The contributions to the decays can be divided into "internal-bremsstrahlung" (IB) and "structure-dependent" (SD) parts in terms of the photon emission. For the IB contribution the photon emits from the $\tau$ lepton as well as external hadron, while the SD one from intermediate states described by the vector and axial-vector form factors, $F_{V, A}$. Our main task is to calculate these form factors which are functions of $t^{2}=(p+q)^{2}$ where $q(p)$ is the four momentum of $\gamma(P)$ and $M_{P}^{2} \leq t^{2} \leq m_{\tau}^{2}$. In general, the momentum dependences of $F_{V, A}$ could also help us to determine the bound state wave functions of mesons.

In this paper, we will use the light front quark model (LFQM) to evaluate the matrix elements in $\tau \rightarrow \nu_{\tau} P \gamma$ decays. The LFQM has been widely applied to study the form factors of weak decays $[1,2,3,4,5,6,7]$. It is the relativistic quark model [8] in which a consistent and relativistic treatment of quark spins and the center-of-mass motion can be carried out. Moreover, the meson state of the definite spins could be relativistically constructed by the Melosh transformation [9]. They are many advantages in the LFQM. For example, the light front wave function is manifestly Lorentz invariant as it is expressed in terms of the momentum fraction variable (in "+" component) in analogy with the parton distributions in the infinite momentum frame. The kinematic subgroup of the light front formalism has the maximum number of interaction-free generators including the boost operator which describes the center-of-mass motion of the bound state [8].

The paper is organized as follows. In Sec. 2, we present the matrix elements and study the form factors in the $P \rightarrow \gamma$ transitions within the framework of the LFQM. We calculate the decay widths of $\tau \rightarrow \nu_{\tau} P \gamma$ in Sec. 3. We also compare our results with those in literature $[10,11]$. We give our conclusions in Sec. 4.

\section{Form Factors in the Light Front Quark Model}

Similar to the radiative meson decay, the decay amplitude for

$$
\tau^{-}(l) \rightarrow \nu_{\tau}(k) P^{-}(p) \gamma(q)
$$


with $P=\pi$ or $K$ can be written as $[10,12,13]$

$$
\begin{aligned}
\mathcal{M}= & \mathcal{M}_{I B}+\mathcal{M}_{S D} \\
\mathcal{M}_{I B}= & G_{F} \cos \theta_{c} e f_{P} m_{\tau} \bar{u}(k)\left(1+\gamma_{5}\right)\left[\frac{p \cdot \epsilon}{p \cdot q}+\frac{q \phi}{2 l \cdot q}-\frac{l \cdot \epsilon}{l \cdot q}\right] v(l), \\
\mathcal{M}_{S D}= & \frac{G_{F} \cos \theta_{c} e}{\sqrt{2}}\left\{i \epsilon_{\mu \nu \rho \sigma} L^{\mu} \epsilon^{\nu} q^{\rho} p^{\sigma} \frac{F_{V}}{M_{P}}\right. \\
& \left.+\bar{u}(k)\left(1+\gamma_{5}\right)[(p \cdot q) \notin-(\epsilon \cdot p) q] v(l) \frac{F_{A}}{M_{P}}\right\}
\end{aligned}
$$

where $L^{\mu}=\bar{u}(k) \gamma^{\mu}\left(1-\gamma_{5}\right) u(l)$ and $F_{A, V}$ are the form-factors corresponding to the vector and axial-vector currents, defined by

$$
\begin{aligned}
\left\langle\gamma(q), P(p)\left|\bar{q} \gamma_{\mu} \gamma_{5} b\right| 0\right\rangle & =e \frac{F_{A}}{M_{P}}\left[(p \cdot q) \epsilon_{\mu}^{*}-\left(\epsilon^{*} \cdot p\right) q_{\mu}\right], \\
\left\langle\gamma(q), P(p)\left|\bar{q} \gamma_{\mu} b\right| 0\right\rangle & =i e \frac{F_{V}}{M_{P}} \varepsilon_{\mu \nu \alpha \beta} \epsilon^{* \nu} q^{\alpha} p^{\beta}
\end{aligned}
$$

with $\epsilon_{\mu}$ being the photon polarization vector and $q(p)$ the four momentum of $\gamma(P)$, respectively. The form factors $F_{A, V}$ in Eq. (4) depend on $t^{2}=(p+q)^{2}$ for which the allowed range is $M_{P}^{2} \leq t^{2} \leq m_{\tau}^{2}$, in contrast to $0 \leq t^{2} \leq M_{P}^{2}$ in $P^{+} \rightarrow l^{+} \nu_{l} \gamma$.

\subsection{Light front formalism}

To calculate of the hadronic matrix elements in Eq. (4), one usually lets $t^{+}=0$ to have a spacelike momentum transfer. However, since the momentum transfer should be always timelike in a real decay process, in this work we use the frame of $t_{\perp}=0$ with the physically accessible kinematic region of $0 \leq t^{2} \leq t_{\max }^{2}$. Within the light front formalism, the meson bound state, which consists of a quark $q_{1}$ and an anti-quark $\bar{q}_{2}$ with the total momentum $p$ and spin $S$, can be written as

$$
\begin{aligned}
\left|M\left(p, S, S_{z}\right)\right\rangle & =\int\left[d k_{1}\right]\left[d k_{2}\right] 2(2 \pi)^{3} \delta^{3}\left(p-k_{1}-k_{2}\right) \\
& \times \sum_{\lambda_{1} \lambda_{2}} \Phi^{S S_{z}}\left(k_{1}, k_{2}, \lambda_{1}, \lambda_{2}\right) b_{q_{1}}^{+}\left(k_{1}, \lambda_{1}\right) d_{\overline{q_{2}}}^{+}\left(k_{2}, \lambda_{2}\right)|0\rangle,
\end{aligned}
$$

where

$$
\begin{aligned}
& {[d k]=\frac{d k^{+} d k_{\perp}}{2(2 \pi)^{3} k^{+}},} \\
& \left\{b_{\lambda^{\prime}}\left(k^{\prime}\right), b_{\lambda}^{\dagger}(k)\right\}=\left\{d_{\lambda^{\prime}}\left(k^{\prime}\right), d_{\lambda}^{\dagger}(k)\right\}=2(2 \pi)^{3} \delta^{3}\left(k^{\prime}-k\right) \delta_{\lambda^{\prime} \lambda},
\end{aligned}
$$

and $k_{1(2)}$ is the on-mass shell light front momentum of the internal quark $q_{1}\left(\bar{q}_{2}\right)$. The light front relative momentum variables $\left(x, k_{\perp}\right)$ are defined by

$$
\begin{aligned}
& k_{1}^{+}=x_{1} p^{+}, \quad k_{2}^{+}=x_{2} p^{+}, \quad x_{1}+x_{2}=1, \\
& k_{1 \perp}=x_{1} p_{\perp}+k_{\perp}, \quad k_{2 \perp}=x_{2} p_{\perp}-k_{\perp} .
\end{aligned}
$$


In the momentum space, the wave function $\Phi^{S S_{z}}$ is given by

$$
\Phi^{S S_{z}}\left(k_{1}, k_{2}, \lambda_{1}, \lambda_{2}\right)=R_{\lambda_{1} \lambda_{2}}^{S S_{z}}\left(x, k_{\perp}\right) \phi\left(x, k_{\perp}\right)
$$

where $\phi\left(x, k_{\perp}\right)$ describes the momentum distribution amplitude of the constituents in the bound state and $R_{\lambda_{1} \lambda_{2}}^{S S_{z}}$ constructs a spin state $\left(S, S_{z}\right)$ out of light front helicity eigenstates $\left(\lambda_{1} \lambda_{2}\right)$, expressed by

$$
R_{\lambda_{1} \lambda_{2}}^{S S_{z}}\left(x, k_{\perp}\right)=\sum_{s_{1}, s_{2}}\left\langle\lambda_{1}\left|\mathcal{R}_{M}^{\dagger}\left(1-x, k_{\perp}, m_{1}\right)\right| s_{1}\right\rangle\left\langle\lambda_{2}\left|\mathcal{R}_{M}^{\dagger}\left(x,-k_{\perp}, m_{2}\right)\right| s_{2}\right\rangle\left\langle\frac{1}{2} s_{1} \frac{1}{2} s_{2} \mid S S_{z}\right\rangle .
$$

In Eq. (9), $\left|s_{i}\right\rangle$ are the Pauli spinors and $\mathcal{R}_{M}$ is the Melosh transformation operator, given by

$$
\mathcal{R}_{M}\left(x, k_{\perp}, m_{i}\right)=\frac{m_{i}+x_{i} M_{0}+i \vec{\sigma} \cdot \vec{k}_{\perp} \times \vec{n}}{\sqrt{\left(m_{i}+x_{i} M_{0}\right)^{2}+k_{\perp}^{2}}}
$$

with

$$
\begin{aligned}
M_{0}^{2} & =\frac{m_{1}^{2}+k_{\perp}^{2}}{x_{1}}+\frac{m_{2}^{2}+k_{\perp}^{2}}{x_{2}}, \\
n & =(0,0,1) .
\end{aligned}
$$

We note that Eq. (9) can, in fact, be expressed as a covariant form [1]

$$
R_{\lambda_{1} \lambda_{2}}^{S S_{z}}\left(x, k_{\perp}\right)=\frac{\sqrt{k_{1}^{+} k_{2}^{+}}}{\sqrt{2} \widetilde{M}_{0}} \bar{u}\left(k_{1}, \lambda_{1}\right) \Gamma v\left(k_{2}, \lambda_{2}\right),
$$

where

$$
\begin{aligned}
& \widetilde{M}_{0} \equiv \sqrt{M_{0}^{2}-\left(m_{1}-m_{2}\right)^{2}}, \\
& \sum_{\lambda} u(k, \lambda) \bar{u}(k, \lambda)=\frac{m+\not k}{k^{+}}, \quad \sum_{\lambda} v(k, \lambda) \bar{v}(k, \lambda)=-\frac{m-\not k}{k^{+}},
\end{aligned}
$$

and

$$
\begin{aligned}
& \Gamma=\gamma_{5} \quad(\text { pseudoscalar, } S=0), \\
& \Gamma=-\not{q}\left(S_{z}\right)+\frac{\hat{\varepsilon} \cdot\left(k_{1}-k_{2}\right)}{M_{0}+m_{1}+m_{2}} \quad(\text { vector }, S=1),
\end{aligned}
$$

with

$$
\begin{aligned}
& \hat{\varepsilon}^{\mu}( \pm 1)=\left[\frac{2}{p^{+}} \vec{\varepsilon}_{\perp}( \pm 1) \cdot \vec{p}_{\perp}, 0, \vec{\varepsilon}_{\perp}( \pm 1)\right], \quad \vec{\varepsilon}_{\perp}( \pm 1)=\mp(1, \pm i) / \sqrt{2} \\
& \hat{\varepsilon}^{\mu}(0)=\frac{1}{M_{0}}\left(\frac{-M_{0}^{2}+p_{\perp}^{2}}{p^{+}}, p^{+}, p_{\perp}\right) .
\end{aligned}
$$


The normalization condition of the meson state is given by

$$
\left\langle M\left(p^{\prime}, S^{\prime}, S_{z}^{\prime}\right) \mid M\left(p, S, S_{z}\right)\right\rangle=2(2 \pi)^{3} p^{+} \delta^{3}\left(\tilde{p}^{\prime}-\tilde{p}\right) \delta_{S^{\prime} S} \delta_{S_{z}^{\prime} S_{z}}
$$

which leads to

$$
\int \frac{d x d^{2} k_{\perp}}{2(2 \pi)^{3}}\left|\phi\left(x, k_{\perp}\right)\right|^{2}=1
$$

In principle, the momentum distribution amplitude $\phi\left(x, k_{\perp}\right)$ can be obtained by solving the light front QCD bound state equation [14, 15]. However, before such first-principle solutions are available, we would have to be contented with phenomenological amplitudes. One example that has been often used in the literature is the Gaussian type wave function:

$$
\phi\left(x, k_{\perp}\right)=N \sqrt{\frac{d k_{z}}{d x}} \exp \left(-\frac{\vec{k}^{2}}{2 \omega_{M}^{2}}\right),
$$

where $N=4\left(\pi / \omega_{M}^{2}\right)^{\frac{3}{4}}, \vec{k}=\left(k_{\perp}, k_{z}\right)$, and $d k_{z} / d x=e_{1} e_{2} / x(1-x) M_{0}$ with $k_{z}$ being defined through

$$
x=\frac{e_{1}-k_{z}}{e_{1}+e_{2}} \quad 1-x=\frac{e_{2}+k_{z}}{e_{1}+e_{2}}, \quad e_{i}=\sqrt{m_{i}^{2}+\vec{k}^{2}}
$$

by

$$
k_{z}=\left(x-\frac{1}{2}\right) M_{0}+\frac{m_{1}^{2}-m_{2}^{2}}{2 M_{0}} .
$$

In particular, with appropriate parameters, the wave function in Eq. (18) describes satisfactorily the pion elastic form factor up to $t^{2} \sim 10 \mathrm{GeV}^{2}[16]$.

\subsection{The Form Factors of $F_{A}$ and $F_{V}$}

The one-loop diagrams that contribute to $F_{V, A}$ are illustrated in Figure 1. From the figures, the hadronic matrix elements in Eq. (4) are found to be

$$
\begin{aligned}
& \left\langle\gamma(q), P(p)\left|\bar{q} \gamma_{\mu}\left(1-\gamma_{5}\right) Q\right| 0\right\rangle=\int \frac{d^{4} k_{1}^{\prime}}{(2 \pi)^{4}} \Lambda_{P} \\
& \times\left\{\gamma_{\mu}\left(1-\gamma_{5}\right) \frac{i\left(k_{1}^{\prime}+m_{Q}\right)}{k_{1}^{\prime 2}-m_{Q}^{2}+i \epsilon} \gamma_{5} \frac{i\left(-k_{2}^{\prime}+m_{q}\right)}{k_{2}^{\prime 2}-m_{q}^{2}+i \epsilon} i e_{q} \phi \frac{i\left(k_{1}^{\prime}+m_{q}\right)}{k_{1}^{2}-m_{q}^{2}+i \epsilon}\right. \\
& \left.-\left(q \leftrightarrow Q, k_{1}^{\prime} \rightarrow k_{2}^{\prime}\right)\right\}
\end{aligned}
$$

where $\Lambda_{P}$ is a vertex function related to $Q \bar{q}$ bound state of the meson $P, k_{2}=p-k_{1}^{\prime}$ and $k_{1}=t-k_{1}^{\prime}=k_{2}+q$. After integrating over the LF momentum $k_{1}^{-}$in Eq. (21), we get

$$
\begin{aligned}
& \left\langle\gamma\left(q_{\gamma}\right), P(p)\left|\bar{q} \gamma_{\mu}\left(1-\gamma_{5}\right) Q\right| 0\right\rangle \\
& =\int_{0}^{p}\left[d^{3} k_{1}^{\prime}\right]\left\{\frac{1}{k_{1}^{-}-k_{1 o n}^{-}}\left(\left.I^{\mu \nu}\right|_{k_{1 o n}^{\prime}-}\right) \frac{\Lambda_{P}}{k_{2}^{\prime-}-k_{2 o n}^{\prime}}-\left(q \leftrightarrow Q, k_{1}^{\prime} \rightarrow k_{2}^{\prime}\right)\right\},
\end{aligned}
$$


where

$$
\begin{aligned}
& {\left[d^{3} k_{1}^{\prime}\right]=\frac{d k_{1}^{+} d k_{1 \perp}}{2(2 \pi)^{3} k_{1}^{\prime+} k_{2}^{\prime+} k_{1}^{+}},} \\
& \left.I^{\mu \nu}\right|_{k_{1 o n}^{-}}=\operatorname{Tr}\left\{\gamma_{\mu}\left(1-\gamma_{5}\right)\left(k_{1}^{\prime}+m_{Q}\right) \gamma_{5}\left(-k_{2}^{\prime}+m_{q}\right) i e_{q} \notin\left(k_{1}+m_{q}\right)\right\} \\
& k_{\text {ion }}^{-}=\frac{m_{i}^{2}+k_{i \perp}^{2}}{k_{i}^{+}}, k_{1(2)}^{\prime-}=p_{o n}^{-}-k_{2(1) o n}^{\prime}, k_{1}^{-}=k_{2 o n}^{-}+q_{\gamma o n}^{-}
\end{aligned}
$$

with $\{$ on $\}$ representing the on-shell particles. By considering the "good" component $\mu=+$, the meson wave function in Eq. (8) and Melosh transformation in Eq. (12) are related to the bound state vertex function $\Lambda_{P}$, given by $[1,17]$ :

$$
\frac{\Lambda_{P}}{k_{2}^{\prime-}-k_{2 o n}^{\prime-}} \rightarrow \frac{\sqrt{k_{1}^{\prime+} k_{2}^{\prime+}}}{\sqrt{2} \widetilde{M}_{0}} \phi\left(x, k_{\perp}\right) \text {. }
$$

Moreover, the matrix elements in Eq. (4) become

$$
\begin{aligned}
\left\langle\gamma(q), P(p)\left|\bar{q} \gamma^{+} \gamma_{5} Q\right| 0\right\rangle & =e \frac{F_{A}}{2 M_{P}}\left(\epsilon_{\perp}^{*} \cdot q_{\perp}\right), \\
\left\langle\gamma(q), P(p)\left|\bar{q} \gamma^{+} Q\right| 0\right\rangle & =-i e \frac{F_{V}}{2 M_{P}} \epsilon^{i j} \epsilon_{i}^{*} q_{j} .
\end{aligned}
$$

Here we have used the LF monentum variables $\left(x, k_{\perp}\right)$ and worked in the frame that the transverse momentum is purely longitudinal, i.e., $t_{\perp}=0$. We note that $t^{2}=t^{+} t^{-} \geq 0$ covers the entire range of momentum transfers. Thus, the relevant quark variables for Figure. 1 are

$$
\begin{aligned}
& k_{1}^{+}=(1+x) q^{+}, \quad k_{2}^{+}=x q^{+}, \quad k_{1 \perp}=(1+x) q_{\perp}-k_{\perp}, \quad k_{2 \perp}=x q_{\perp}-k_{\perp} . \\
& k_{1}^{\prime+}=\left(1-x^{\prime}\right) p^{+}, \quad k_{2}^{\prime+}=x^{\prime} p^{+}, \quad k_{1 \perp}^{\prime}=-\left(1-x^{\prime}\right) q_{\perp}+k_{\perp}^{\prime}, \quad k_{2 \perp}^{\prime}=-x^{\prime} q_{\perp}-k_{\perp}^{\prime}
\end{aligned}
$$

where $x^{\prime}(x)$ is the momentum fraction of the anti-quark in the meson (photon) state. At the quark loop, it requires that

$$
k_{2(1)}^{\prime+}=k_{2}^{+}, \quad k_{2(1) \perp}^{\prime}=k_{2 \perp},
$$

for Figure 1a(b). Therefore, the trace $I^{\mu \nu}$ in Eq. (23) can be easily carried out. The form factors $F_{A}$ and $F_{V}$ in Eq. (25) are then found to be

$$
\begin{aligned}
F_{A}\left(t^{2}\right)= & -4 M_{P} \int_{0}^{\xi} \frac{d x d^{2} k_{\perp}}{2(2 \pi)^{3} \xi}\left\{\frac{2(1+2 x)\left(x+x^{\prime}\right)}{3} \frac{m_{q}-x^{\prime}\left(m_{Q}-m_{q}\right) k_{\perp}^{2} \Theta}{m_{q}^{2}+k_{\perp}^{2}} \frac{\Phi\left(x^{\prime}, k_{\perp}^{2}\right)}{x^{\prime}\left(1-x^{\prime}\right)} 28\right) \\
& \left.+\frac{(1+2 x)\left(1-x^{\prime \prime}+x\right)}{3} \frac{m_{Q}+\left(1-x^{\prime \prime}\right)\left(m_{Q}-m_{q}\right) k_{\perp}^{2} \Theta}{m_{Q}^{2}+k_{\perp}^{2}} \frac{\Phi\left(x^{\prime \prime}, k_{\perp}^{2}\right)}{x^{\prime \prime}\left(1-x^{\prime \prime}\right)}\right\}, \\
F_{V}\left(t^{2}\right)= & -4 M_{P} \int_{0}^{\xi} \frac{d x d^{2} k_{\perp}}{2(2 \pi)^{3} \xi}\left\{\frac{2\left(x+x^{\prime}\right)}{3} \frac{(1+2 x) m_{q}-A k_{\perp}^{2} \Theta}{m_{q}^{2}+k_{\perp}^{2}} \frac{\Phi\left(x^{\prime}, k_{\perp}^{2}\right)}{x^{\prime}\left(1-x^{\prime}\right)}\right. \\
& \left.+\frac{\left(1-x^{\prime \prime}+x\right)}{3} \frac{(1+2 x) m_{Q}-B k_{\perp}^{2} \Theta}{m_{Q}^{2}+k_{\perp}^{2}} \frac{\Phi\left(x^{\prime \prime}, k_{\perp}^{2}\right)}{x^{\prime \prime}\left(1-x^{\prime \prime}\right)}\right\},
\end{aligned}
$$


respectively, where

$$
\begin{aligned}
\xi & =\frac{p^{+}}{q^{+}}=\frac{M_{p}^{2}}{t^{2}-M_{p}^{2}}, \\
A & =x^{\prime}\left(m_{Q}-m_{q}\right)-2 x m_{q} \\
B & =-\left(1-x^{\prime \prime}\right)\left(m_{Q}-m_{q}\right)-2 x m_{q}, \\
\Phi\left(x, k_{\perp}^{2}\right) & =N\left(\frac{x(1-x)}{2\left(M_{0}^{2}-\left(m_{Q}-m_{q}\right)^{2}\right)}\right)^{1 / 2} \sqrt{\frac{d k_{z}}{d x}} \exp \left(-\frac{\vec{k}^{2}}{2 \omega_{M}^{2}}\right) \\
\Theta & =\frac{1}{\Phi\left(x, k_{\perp}^{2}\right)} \frac{d \Phi\left(x, k_{\perp}^{2}\right)}{d k_{\perp}^{2}} \\
x^{\prime} & =x\left(\frac{t^{2}-M_{p}^{2}}{M_{p}^{2}}\right), x^{\prime \prime}=1-x\left(\frac{t^{2}-M_{p}^{2}}{M_{p}^{2}}\right), \vec{k}=\left(\vec{k}_{\perp}, \vec{k}_{z}\right) .
\end{aligned}
$$

\section{Decay widths}

To compute numerical values of the form factors, we use $f_{\pi}=0.925, m_{Q}=m_{q}=m_{u}=$ $m_{d}=0.25, M_{\pi}=0.14, \omega_{\pi}=0.3$ for the $\pi$ meson, and $f_{K}=0.113, m_{Q}=m_{s}=0.4$, $m_{q}=m_{u}=0.25, M_{K}=0.495, \omega_{K}=0.37$ for the $K$ meson in GeV [18, 19], respectively. We start with the decay of $\tau \rightarrow \nu_{\tau} \pi \gamma$. We define $x=2 l \cdot q / m_{t}^{2}$ and $y=2 l \cdot p / m_{t}^{2}$. In the $\tau$ rest frame, $x(y)$ corresponds to the photon (pion ) energy of $E_{\gamma(\pi)}$, expressed in units of $m_{\tau} / 2$ as

$$
x=\frac{2 E_{\gamma}}{m_{\tau}} \quad \text { and } \quad y=\frac{2 E_{\pi}}{m_{\tau}} .
$$

In terms of $x$ and $y$, one has the following kinematics:

$$
\begin{aligned}
p \cdot q & =\frac{m_{\tau}^{2}}{2}(x+y-1-r), \\
t^{2} & =(s-k)^{2}=(p+q)^{2}=m_{\tau}^{2}(x+y-1) .
\end{aligned}
$$

The physical allowed regions for $x$ and $y$ are given by:

$$
\begin{aligned}
0 & \leq x \leq 1-r, \\
1-x+\frac{r}{1-x} & \leq y \leq 1+r,
\end{aligned}
$$

with

$$
r=\left(\frac{m_{\pi}}{m_{\tau}}\right)^{2} \sim 6.21 \times 10^{-3}
$$

where we have used $m_{\tau}=1.777 \mathrm{GeV}$. We now calculate the differential decay rate of $\tau^{-} \rightarrow \nu_{\tau} \pi^{-} \gamma$, given by

$$
d \Gamma\left(\tau \rightarrow \nu_{\tau} \pi \gamma\right)=\frac{1}{2 m_{\tau}} \delta^{(4)}(l-q-p-k)|\mathcal{M}|^{2} \frac{d \vec{q}}{(2 \pi)^{3} 2 E_{\gamma}} \frac{d \vec{p}}{(2 \pi)^{3} 2 E_{\pi}} \frac{d \vec{k}}{(2 \pi)^{3} 2 E_{\nu}}
$$


In the tau rest frame, one has

$$
\frac{d^{2} \Gamma}{d x d y}=\frac{m_{\tau}}{256 \pi^{3}}|\mathcal{M}|^{2},
$$

where

$$
|\mathcal{M}|^{2}=\left|\mathcal{M}_{I B}\right|^{2}+\left|\mathcal{M}_{S D}\right|^{2}+2 \operatorname{Re}\left(\mathcal{M}_{I B} \mathcal{M}_{S D}^{*}\right) .
$$

By writing the decay width $\Gamma$ in terms of the three different source as in Eq. (38), i.e.,

$$
\Gamma_{\text {total }}=\Gamma_{I B}+\Gamma_{S D}+\Gamma_{I N T}
$$

and the non-radiative decay $\left(\tau^{-} \rightarrow \nu_{\tau} \pi^{-}\right)$width

$$
\Gamma_{\tau \rightarrow \nu_{\tau} \pi}=\frac{G_{F}^{2}\left|V_{u d}\right|^{2} \theta_{c} f_{\pi}^{2}}{8 \pi} m_{\tau}^{3}(1-r)^{2}=2.44 \times 10^{-10} \mathrm{MeV},
$$

we obtain that

$$
\begin{aligned}
\frac{d^{2} \Gamma_{I B}}{d x d y}= & \frac{\alpha}{2 \pi} \rho_{I B}(x, y) \frac{\Gamma_{\tau \rightarrow \nu_{\tau} \pi}}{(1-r)^{2}} \\
\frac{d^{2} \Gamma_{S D}}{d x d y}= & \frac{\alpha}{16 \pi} \frac{m_{\tau}^{4}}{f_{\pi}^{2} m_{\pi}^{2}}\left[\left|F_{V}\right|^{2} \rho_{V V}(x, y)\right. \\
& \left.+2 \operatorname{Re}\left(F_{V} F_{A}^{\star}\right) \rho_{V A}(x, y)+\left|F_{A}\right|^{2} \rho_{A A}(x, y)\right] \frac{\Gamma_{\tau \rightarrow \nu_{\tau} \pi}}{(1-r)^{2}}, \\
\frac{d^{2} \Gamma_{I N T}}{d x d y}= & \frac{\alpha}{2 \sqrt{2} \pi} \frac{m_{\tau}^{2}}{f_{\pi} m_{\pi}}\left[\rho_{I N T V}(x, y) \operatorname{Re}\left(F_{V}\right)+\rho_{I N T A}(x, y) \operatorname{Re}\left(F_{A}\right)\right] \frac{\Gamma_{\tau \rightarrow \nu_{\tau} \pi}}{(1-r)^{2}}
\end{aligned}
$$

where

$$
\begin{aligned}
\rho_{I B}(x, y) & =\frac{\left[r^{2}(x+2)-2 r(x+y)+(x+y-1)\left(x^{2}-3 x+2+x y\right)\right](r-y+1)}{(r-x-y+1)^{2} x^{2}}, \\
\rho_{V V}(x, y) & =-\left[r^{2}(x+y)+2 r(1-y)(x+y)+(x+y-1)\left(x^{2}+y^{2}-x-y\right)\right], \\
\rho_{A A}(x, y) & =\rho_{V V}(x, y, r), \\
\rho_{V A}(x, y) & =\left[r^{2}(x+y)+(1-x-y)(y-x)\right]\left(r^{2}-x-y+1\right), \\
\rho_{I N T A}(x, y) & =-\frac{(r-x-y+1)(r-y+1)}{x} \\
\rho_{I N T V}(x, y) & =\frac{\left[r^{2}-2 r(x+y)+(1-x+y)(x+y-1)\right](r-y+1)}{(r-x-y+1) x} .
\end{aligned}
$$

To simplify our calculations, we now introduce $\lambda$ as a new parameter

$$
\lambda=\frac{t^{2}}{m_{\tau}^{2}}=x+y-1 .
$$


The kinematical boundaries for $x$ and $\lambda$ are given by:

$$
\begin{aligned}
\lambda-r & \leq x \leq 1-\frac{r}{\lambda}, \\
r & \leq \lambda \leq 1 .
\end{aligned}
$$

By integrating the variable $x$ in the phase space, from Eq. (41), we derive the expressions of differential decay widths for the invariant mass spectrum as:

$$
\begin{aligned}
\frac{d \Gamma_{I B}}{d \lambda}= & \frac{\alpha}{2 \pi}\left[(1-\lambda)\left(r^{2}+2 r \lambda-4 \lambda+\lambda^{2}\right)\right. \\
& \left.+\left(r^{2} \lambda+2 r \lambda-2 \lambda-2 \lambda^{2}+\lambda^{3}\right) \ln \lambda\right] \frac{1}{\lambda^{2}-r \lambda} \frac{\Gamma_{\tau \rightarrow \nu_{\tau} \pi}}{(1-r)^{2}}, \\
\frac{d \Gamma_{S D}}{d \lambda}= & \frac{\alpha}{48 \pi} \frac{m_{\tau}^{4}}{f_{\pi}^{2} m_{\pi}^{2}} \frac{(\lambda-1)^{2}(\lambda-r)^{3}(1+2 \lambda)}{\lambda^{2}}\left(\left|F_{V}\right|^{2}+\left|F_{A}\right|^{2}\right) \frac{\Gamma_{\tau \rightarrow \nu_{\tau} \pi}}{(1-r)^{2}}, \\
\frac{d \Gamma_{I N T V}}{d \lambda}= & \frac{\alpha}{2 \sqrt{2} \pi} \frac{m_{\tau}^{2}}{f_{\pi} m_{\pi}} \frac{(\lambda-r)^{2}(1-\lambda+\lambda \ln \lambda)}{\lambda} \operatorname{Re}\left(F_{V}\right) \frac{\Gamma_{\tau \rightarrow \nu_{\tau} \pi}}{(1-r)^{2}}, \\
\frac{d \Gamma_{I N T A}}{d \lambda}= & \frac{\alpha}{2 \sqrt{2} \pi} \frac{m_{\tau}^{2}}{f_{\pi} m_{\pi}}[(1-\lambda)(r-2 \lambda-1) \\
& \left.+\left(r \lambda-2 \lambda-\lambda^{2}\right) \ln \lambda\right] \frac{\lambda-r}{\lambda} \operatorname{Re}\left(F_{A}\right) \frac{\Gamma_{\tau \rightarrow \nu_{\tau} \pi}}{(1-r)^{2}} .
\end{aligned}
$$

Note that for the IB part in Eqs. (40) and (44) the contribution is infrared divergent when $x$ closed to 0 and, moreover, there is also an enhancement in the limit $\lambda \rightarrow r$. This means that the IB term contains the logarithm divergent as $t^{2} \rightarrow m_{\pi}^{2}$. To obtain the decay width of $\tau \rightarrow \nu_{\tau} \pi \gamma$, a cut on the photon energy is needed. The differential decay width $d \Gamma_{I B} / d \lambda$ as a function of $\lambda=t^{2} / m_{\tau}^{2}$ in terms of $\Gamma_{\tau \rightarrow \nu_{\tau} \pi}$ is shown in Figures 2 and 3 with two different cuts of $E_{\gamma}=50$ and $400 \mathrm{MeV}$, respectively. In Table 1, we list the integrated decay width ratio of $R_{\pi}=\Gamma_{\tau \rightarrow \nu_{\tau} \pi \gamma} / \Gamma_{\tau \rightarrow \nu_{\tau} \pi}$ for the two cuts. As shown in Table

Table 1: Integrated Decay width ratio for $\tau \rightarrow \nu_{\tau} \pi \gamma$.

\begin{tabular}{|c|c|c|c|c|c|c|}
\hline Integrated decay width ratio & IB & SD & INT & Sum & Ref.[10] & Ref.[11] \\
\hline \hline $10^{-3} R_{\pi}\left(E_{\gamma} \geq 50\right) \mathrm{MeV}$ & 13.1 & 1.48 & 1.61 & 16.2 & - & 14.8 \\
\hline $10^{-3} R_{\pi}\left(E_{\gamma} \geq 400\right) \mathrm{MeV}$ & 1.48 & 1.48 & 0.90 & 3.86 & 1.16 & 2.76 \\
\hline
\end{tabular}

1, our results for the decay width of $\tau \rightarrow \nu_{\tau} \pi \gamma$ are larger than those in Refs. [10] and

[11], respectively, due to our bigger SD contribution. From Table 1, it is interesting to see that $\Gamma_{I N T}$ which depends on the photon energy cut can be as large as $\Gamma_{S D}$.

For the decay of $\tau \rightarrow \nu_{\tau} K \gamma$, we use $\Gamma_{\tau \rightarrow \nu_{\tau} K}=1.759 \times 10^{-11} \mathrm{MeV}$. The differential decays width for $\Lambda=t^{2} / m_{\tau}^{2} \geq 0.2 \mathrm{GeV}$ in terms of $\Gamma_{\tau \rightarrow \nu_{\tau} K}$ is plotted in Figure 4. From 
the figure, we see that the SD contribution to the decay width is dominant for values of $t^{2}$. The reason is that the large kaon mass suppresses the IB contribution. In Table 2, we show the decay width ratio of $R_{K}=\Gamma_{\tau \rightarrow \nu_{\tau} K \gamma} / \Gamma_{\tau \rightarrow \nu_{\tau} K}$ with two different invariant mass cuts of $t=800$ and $1200 \mathrm{MeV}$. Form Table 2, we find that the results in Ref. [11] are

Table 2: Integrated decay width ratio for $\tau \rightarrow \nu_{\tau} K \gamma$.

\begin{tabular}{|c|c|c|c|c|c|}
\hline Integrated Decay width ratio & IB & SD & INT & Sum & Ref.[11] \\
\hline \hline $10^{-4} R_{K}(t \geq 800 \mathrm{MeV})$ & 6.51 & 7.36 & 5.26 & 19.13 & 35.8 \\
\hline $10^{-4} R_{K}(t \geq 1200 \mathrm{MeV})$ & 0.72 & 3.80 & 0.86 & 5.38 & 9.1 \\
\hline
\end{tabular}

twice as large as our predictions.

We now study the ratio

$$
R=\frac{\sum_{P=\pi, K} B r\left(\tau \rightarrow \nu_{\tau} P\right)}{B r\left(\tau \rightarrow e \nu_{e} \bar{\nu}_{\tau}\right)}
$$

and examine both theoretical and experimental values. In the standard model, the ratio is given by:

$$
R^{S M}=0.646
$$

while the recent experimental average is [18]

$$
R^{e x p}=0.66100 \pm 0.00725 \text {. }
$$

Clearly, there is a discrepancy between $R^{S M}$ and $R^{e x p}$. However, it is believed that it arises from the radiative corrections. At $O(\alpha)$, the radiative corrected decay width is found to be

$$
\Gamma\left(\tau^{-} \rightarrow \nu_{\tau} \pi^{-}(\gamma)\right)=\Gamma\left(\tau^{-} \rightarrow \nu_{\tau} \pi^{-}\right)+\Gamma\left(\tau^{-} \rightarrow \nu_{\tau} \pi^{-} \gamma\right) \sim 2.48 \times 10^{-10} \mathrm{MeV}
$$

with $E_{\gamma} \geq 50 \mathrm{MeV}$. Similarly, for the mode with $K$, one has that

$$
\Gamma\left(\tau^{-} \rightarrow \nu_{\tau} K^{-}(\gamma)\right) \sim 1.61 \times 10^{-11} \mathrm{MeV}
$$

with the same photon energy cut as the pion mode. In the standard model, the radiative corrected width of $\tau \rightarrow e \nu \bar{\nu}[20]$ is given by

$$
\Gamma\left(\tau^{-} \rightarrow e^{-} \nu_{e} \bar{\nu}_{\tau}(\gamma)\right)=(4.033 \pm 0.005) \times 10^{-10} \mathrm{MeV}
$$

From the Eq. (48)-(50), we obtain

$$
R^{\text {Theor, } O(\alpha)} \sim 0.655
$$

which agrees with experimental data in Eq. (47) within the errors. 


\section{Conclusions}

We have studied the decays of $\tau \rightarrow \nu_{\tau} \pi(K) \gamma$ in the light front quark model. We have calculated the form factors and used them to evaluate the decay widths. We have

found that, in the standard model, the decay widths are $1.62 \times 10^{-2}\left(3.86 \times 10^{-3}\right) \Gamma_{\tau \rightarrow \nu_{\tau} \pi}$ and $1.91 \times 10^{-3}\left(5.38 \times 10^{-4}\right) \Gamma_{\tau \rightarrow \nu_{\tau} K}$ with the cuts of $E_{\gamma}=50\left(E_{\gamma}=400\right) \mathrm{MeV}$ and $t_{0}=800(1200) \mathrm{MeV}$ for $\tau \rightarrow \nu_{\tau} \pi \gamma$ and $\tau \rightarrow \nu_{\tau} K \gamma$, respectively. We have also shown that with including the radiative decay widths, the experimental rate for $\tau \rightarrow \nu_{\tau}(\pi, K)$ can be understood. In future, as the tau-charm factories will produce a large number of samples of $\tau$ lepton pairs, these rare $\tau$ decays should be precisely measured and thus the structure-dependent form-factors can be well tested. In this case, the decays can be used to probe new physics similar to $K_{l 3}[21]$ and $K_{l 2 \gamma}$ decays [22].

\section{Acknowledgments}

This work was supported in part by the National Science Council of the Republic of China under the Grant Nos. NSC91-2112-M-007-043 and NSC91-2112-M-007-046. 


\section{References}

[1] W. Jaus, Phys. Rev. D41, 3394 (1990); 44, 2851 (1991).

[2] P.J. O'Donnell and Q.P. Xu, Phys. Lett. B325, 219 (1994).

[3] P.J. O’Donnell, Q.P. Xu, and H.K.K. Tung, Phys. Rev. D52, 3966 (1995).

[4] C.Y. Cheung, C.W. Hwang, and W.M. Zhang, Z. Phys. C75, 657 (1997).

[5] Hai-Yang Cheng, Chi-Yee Cheung and Chien-Wen Hwang, Phys. Rev. D55, 1559 (1997).

[6] C. Q. Geng, C. C. Lih and W. M. Zhang, Phys. Rev. D57 5697(1998).

[7] C. C. Lih, C. Q. Geng and W. M. Zhang, Phys. Rev. D59 (1999) 114002.

[8] Also see a review by W. M. Zhang, Chin. J. Phys. 31, 717 (1994).

[9] H. J. Melosh, Phys. Rev. D9 (1974) 1095.

[10] Sreeparna Banerjee, Phys. Rev. D34, 2080 (1986).

[11] R. Decker and M. Finkemeier, Phys. Rev. D 48, 4203 (1993) [Addendum-ibid. D 50, 7079 (1994)]

[12] J. Kim and L. Resenick, Phys. Rev. D48 (1980) 1330.

[13] S. G. Brown and S. A. Bludman, Phys. Rev. 136, B1160 (1964); P. de Baenst and J. Pestieau, Nuovo Cimento 53A, 407 (1968).

[14] K. G. Wilson, T. Walhout, A. Harindranath, W. M. Zhang, R. J. Perry and S. Glazek Phys.Rev. D49 (1994) 6720-6766

[15] W. M. Zhang, Phys. Rev. D56 (1997) 1528.

[16] P.L. Chung, F. Coester, and W.N. Polyzou, Phys. Lett. B205, 545 (1988).

[17] Demchuk et al., Phys. Atom. Nucl 59, 2152 (1996).

[18] Particle Data Group, Phys. Rev. D66, Part I (2002).

[19] B. R. Holstein, Phys. Lett. B244, 83 (1990). 
[20] William J. Marciano and A. Sirlin, Phys. Rev. Lett. 61, 1815 (1988).

[21] G. Belanger and C. Q. Geng, Phys. Rev. D 44, 2789 (1991).

[22] C. Q. Geng and S. K. Lee, Phys. Rev. D 51, 99 (1995) [arXiv:hep-ph/9410347];

C. H. Chen, C. Q. Geng and C. C. Lih, Phys. Rev. D 56, 6856 (1997) [arXiv:hepph/9709447]. 


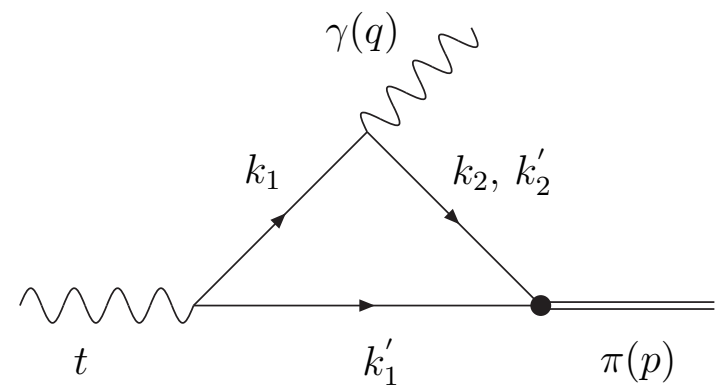

(a)

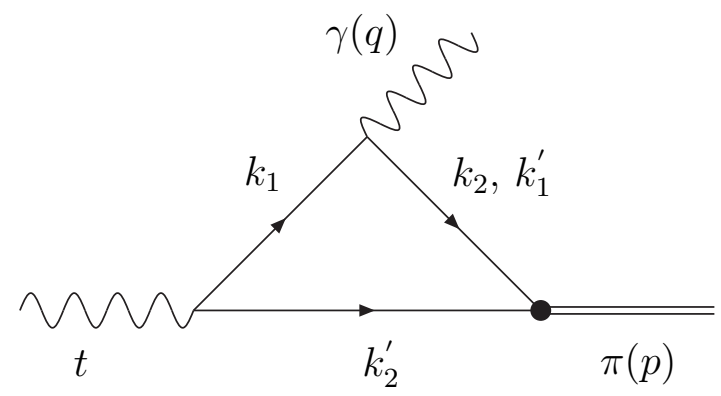

(b)

Figure 1: Loop diagrams that contribute $\tau \rightarrow \nu_{\tau} \pi \gamma$. 


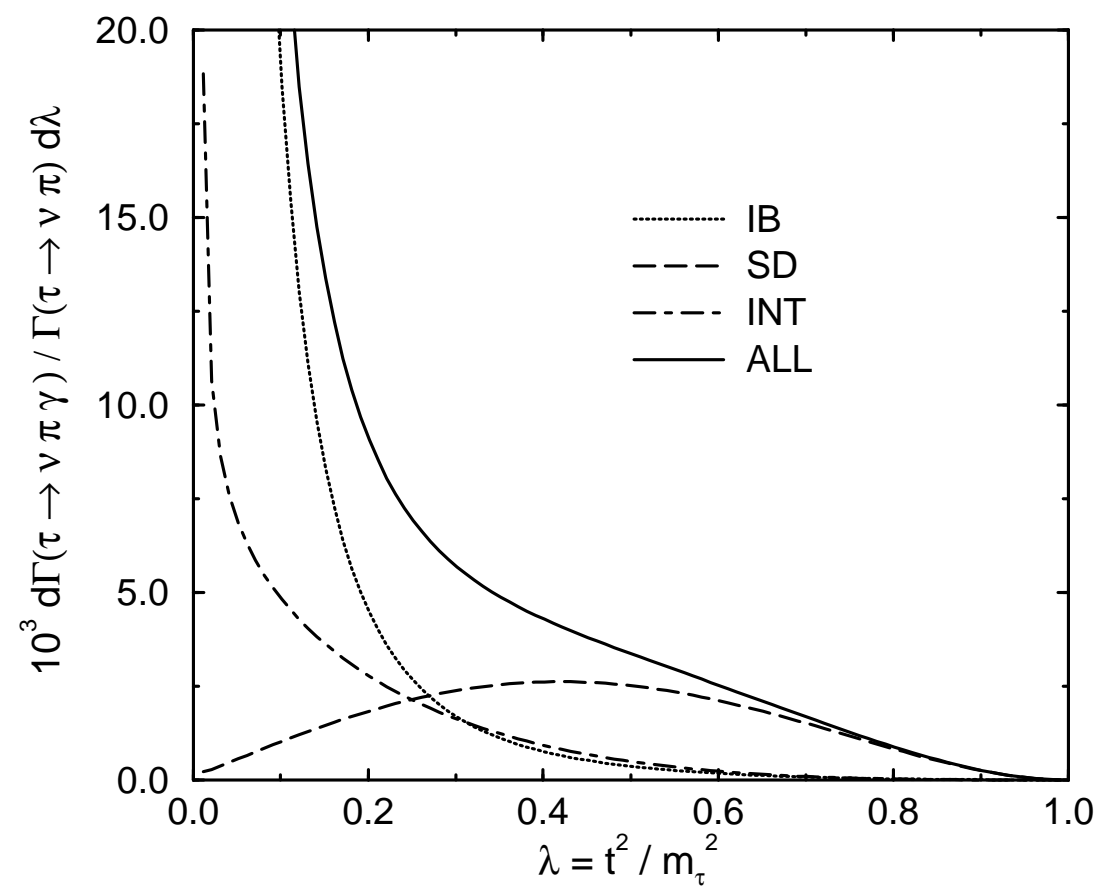

Figure 2: Differential decay width of $\Gamma\left(\tau \rightarrow \nu_{\tau} \pi \gamma\right)$ in terms of $\Gamma_{\tau \rightarrow \nu_{\tau} \pi}$ as a function of $\lambda=t^{2} / m_{\tau}^{2}$ with $E_{\gamma} \geq 50 \mathrm{MeV}$. The dot, dash, dash-dot and solid curves stand for the contributions of IB, SD, INT, and total parts, respectively. 


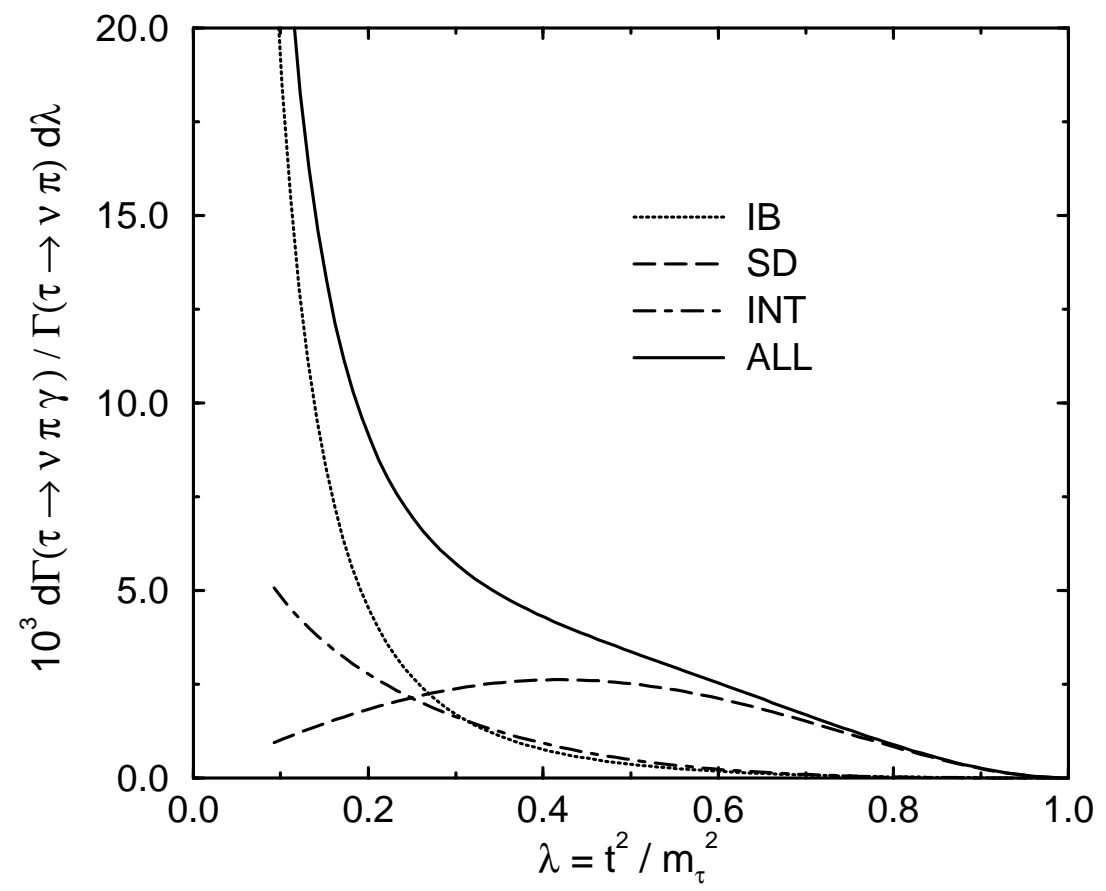

Figure 3: Same as Figure 2 but with $E_{\gamma} \geq 400 \mathrm{MeV}$. 


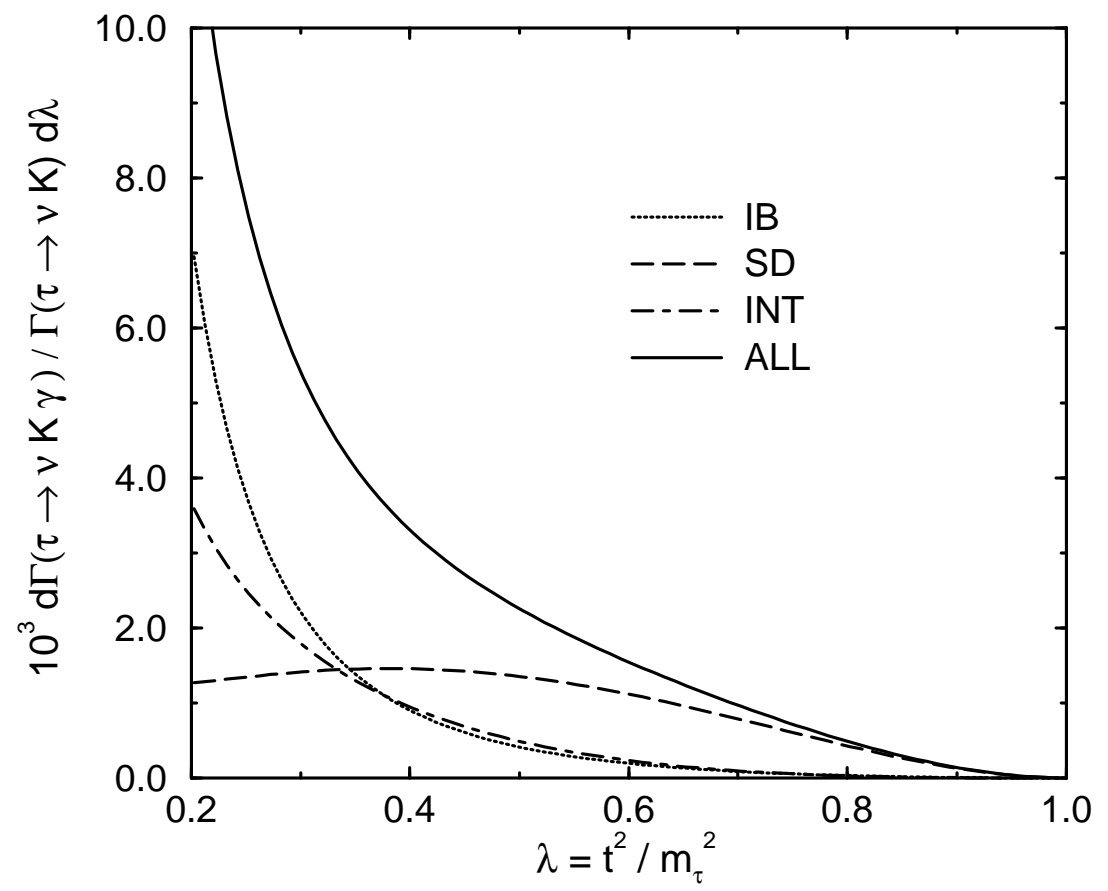

Figure 4: Differential decay width of $\Gamma\left(\tau \rightarrow \nu_{\tau} K \gamma\right)$ as a function of $\lambda=t^{2} / m_{\tau}^{2}$ in terms of $\Gamma_{\tau \rightarrow \nu_{\tau} K}$. Caption is the same as in Figure 2. 